\title{
Eclipsing binaries hiding in the background: the Kepler Pixel Project
}

\author{
A. Forró ${ }^{1,2,3}$ and R. Szabó ${ }^{1,2}$ \\ 1 Konkoly Thege Miklós Astronomical Institute, Research Centre for \\ Astronomy and Earth Sciences, (E-mail: forro.adrienn@csfk.mta.hu) \\ ${ }^{2}$ MTA CSFK Lendület Near-Field Cosmology Research Group \\ 3 Eötvös Loránd University, Department of Astronomy
}

Received: October 31, 2019; Accepted: January 20, 2020

\begin{abstract}
The aim of the Kepler Pixel Project is to discover new pulsating and other types of variable stars in the individual pixels of the original Kepler mission. In the framework of the project, 1272 eclipsing binary candidates were identified in the background pixels. After eliminating false positives and those stars that are already present in the Kepler Eclipsing Binary Catalog, we were left with 776 new eclipsing binaries. This is a substantial and significant addition to the 2922 eclipsing binaries present in the catalog. We present the methods we applied, examine the 4-year (Q1-Q17) light curves of selected newly found variable stars. The applied methods are automatic, therefore they can be used in the future to explore the vast amounts of data produced by other space missions (e.g. TESS, later PLATO).
\end{abstract}

Key words: binaries - data analysis - photometry - Kepler

\section{Introduction}

During its original mission, the Kepler space telescope measured the brightness of more than 160000 stars, producing a quasi-continuous 4-year-long run of observational data with unprecedented photometric precision. Targets investigated so far are mainly those listed in the Kepler Input Catalog (Brown et al., 2011), but more findings indicate that even the background pixels of Kepler's data hold interesting new information waiting to be mined.

\section{The Kepler Pixel Project}

The Kepler Pixel Project (Szabó, 2018) sets the aim of discovering new pulsating and other type of variable stars in the original Kepler field. Since Q4 was a relatively quiescent observing period, we decided to start our search using this dataset.

We extracted each individual pixel of the long-cadence $(30 \mathrm{~min})$ files, which resulted in more than 6 million light curves. All pixels were examined, regardless of whether or not they belong to the main target. The initial goal was to 
look for faint background RR Lyrae stars, so we specified the filtering criteria accordingly. Our potential candidate list includes pixels that show periods between 0.25 and 1 day and have Fourier spectra with at least two harmonics of the main frequency with decreasing amplitude.

\section{Results}

The aforementioned criteria yielded $\sim 12500$ candidate pixels. However, one pixel does not equal one candidate; in the majority of cases a couple of pixels are available for each candidate. Despite our specific criteria, $~ 90 \%$ of our candidates are eclipsing binary stars. We successfully identified 1272 target pixel files containing an eclipsing binary candidate, in most cases located in the background.

Since the goal was to find new variable stars in the field, those candidates that are already listed in the Kepler Eclipsing Binary Catalog (Prša et al., 2011; Abdul-Masih et al., 2016) were excluded. This cross-match left us with 777 new candidates. One of our candidate's light curve was in fact the result of contamination by a bright nearby star, consequently it was removed from our candidate list as well. The final list of potentially new eclipsing binaries in the original Kepler-field has 776 candidates, which consist of 2778 individual pixels in total. In Fig. 1 and Fig. 2, typical candidate light curves are shown.

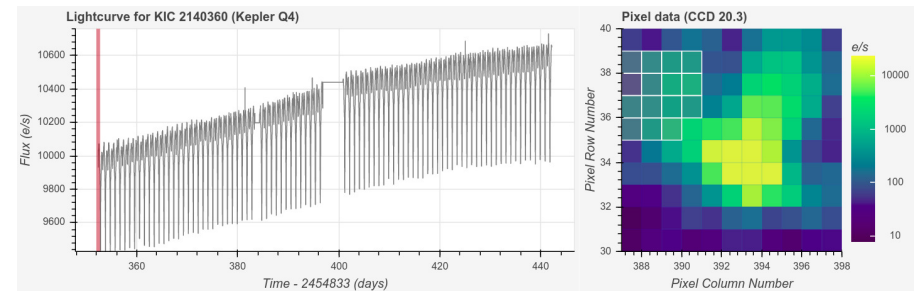

Figure 1. The Q4 light curve of the candidate found in the background of the main target KIC 2140360. The pixels containing the candidate are marked with white edges.

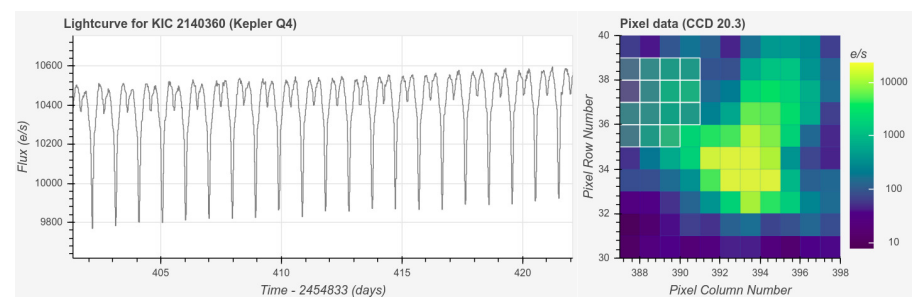

Figure 2. A 10-day-long zoom-in of the Fig. 1 Q4 lighcurve. 
After identifying the Q4 candidates, their data from all the other quarters weer extracted. There were cases when the targets could not be measured in a particular quarter. For example, it is unfortunately quite common that the background pixels containing the candidate are located near the edge of the target pixel file, so in some quarters the candidates might be outside the image.

In the majority of the cases, however, the 4-year nearly continuous light curve was successfully created by stitching together the data of all 17 quarters. The 4-year light curves were stitched for each individual pixels automatically, then the pixels belonging to one candidate were summed.

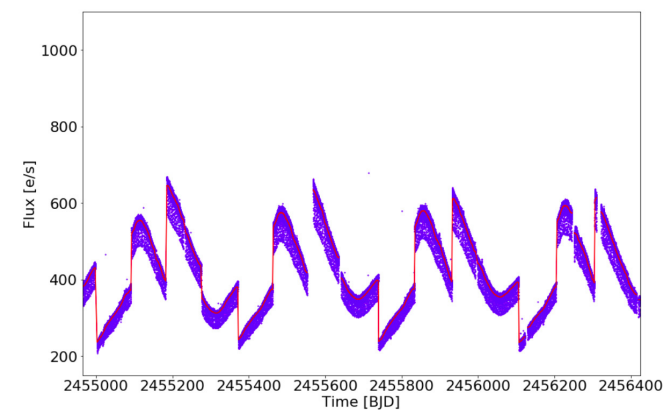

Figure 3. The Q1-Q17 light curve of the candidate found in the background of KIC 2140360. The different trends are quite apparent from quarter to quarter. The red line indicates the trend fitted with the Wötan code.

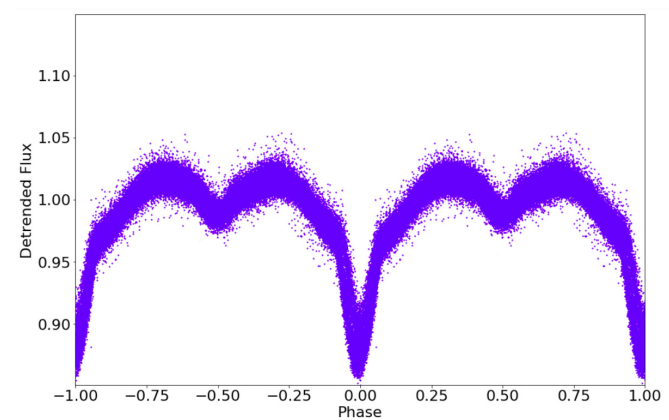

Figure 4. The folded light curve of a candidate with period 0.975 day, after de-trending.

Individual pixels almost always show long-term trends. Sometimes even adding up all candidate pixels doesn't remove the trends, only reduces them. 
Moreover, trends change from quarter to quarter, therefore it is crucial to remove them in order to avoid uneven light curves. To remove these trends, we used the Wötan (Hippke et al., 2019) Python code. Figure 3 shows the stitched light curve for the candidate in the background of KIC 2140360, the folded light curve is seen in Fig. 4. The project is still ongoing, the first results will be published in more detail in a separate paper.

\section{Summary}

In the Kepler Pixel Project, by investigating each individual pixel of the original Kepler mission Q4 quarter images, we managed to find 1272 eclipsing binary candidates, and 776 of them turned out to be new (i.e., not in the Kepler Eclipsing Binary Catalog) candidates. We obtained the data from the other quarters as well, thus creating 4-year-long light curves. Kepler's unprecedented photometric precision enables us to investigate the discovered systems in more detail. The algorithms developed for the project can be used for exploring other space mission data (e.g. TESS, later PLATO).

Acknowledgements. The research leading to these results has received funding from the LP2018-7/2019 Lendlet grant of the Hungarian Academy of Sciences and from the NKFIH K-115709 grant of the National Research, Development and Innovation Office of Hungary. This research made use of Lightkurve, a Python package for Kepler and TESS data analysis (Lightkurve Collaboration et al., 2018).

\section{References}

Abdul-Masih, M., Prša, A., Conroy, K., et al., Kepler Eclipsing Binary Stars. VIII. Identification of False Positive Eclipsing Binaries and Re-extraction of New Light Curves. 2016, Astron. J., 151, 101, DOI: 10.3847/0004-6256/151/4/101

Brown, T. M., Latham, D. W., Everett, M. E., \& Esquerdo, G. A., Kepler Input Catalog: Photometric Calibration and Stellar Classification. 2011, Astron. J., 142, 112, DOI: $10.1088 / 0004-6256 / 142 / 4 / 112$

Hippke, M., David, T. J., Mulders, G. D., \& Heller, R., Wōtan: Comprehensive Time-series Detrending in Python. 2019, Astron. J., 158, 143, DOI: 10.3847/1538$3881 /$ ab3984

Lightkurve Collaboration, Cardoso, J. V. d. M., Hedges, C., et al. 2018, Lightkurve: Kepler and TESS time series analysis in Python, Astrophysics Source Code Library

Prša, A., Batalha, N., Slawson, R. W., et al., Kepler Eclipsing Binary Stars. I. Catalog and Principal Characterization of 1879 Eclipsing Binaries in the First Data Release. 2011, Astron. J., 141, 83, DOI: 10.1088/0004-6256/141/3/83

Szabó, R., The Kepler Pixel Project. in , The RR Lyrae 2017 Conference. Revival of the Classical Pulsators: from Galactic Structure to Stellar Interior Diagnostics, ed. R. Smolec, K. Kinemuchi, \& R. I. Anderson, Vol. 6, 119-123 